\title{
EDEMA PULMONAR UNILATERAL DE LAS GRANDES ALTURAS: PRESENTACIÓN DE UN CASO CLÍNICO
}

Diara Avila Reges Angela Maria Girdido Marioyas

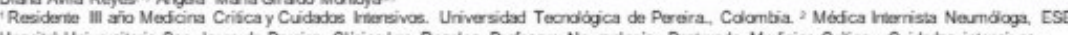

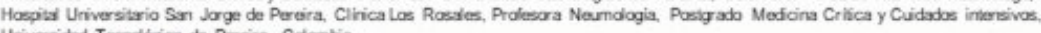

Unversidad Tecrobióica de Pereira. Colombia

\section{INTRODUCCIÓN}

El edema pulmonar de las grandes alturas (EPGA) es una condición clínica potencialmente mortal, que se presenta típicamente posterior al ascenso rápido a grandes altitudes sin previo aclimatamiento y depende de la altitud absoluta alcanzada y la velocidad de ascenso. Es causado por un aumento exagerado de la presión arterial pulmonar y un aumento de la permeabilidad vascular pulmonar posterior a la hipoxia alveolar. (1,2) El umbral de $2500 \mathrm{~m}$ s.n.m. es suficiente para presentar enfermedades relacionadas con la altura, y la sintomatología se desarrolla en los primeros 2 días después de la exposición a altitudes superiores a 3000 m s.n.m. (2.3) El EPGA tiene un espectro clínico variable y según la severidad del cuadro puede progresar hacia la insuficiencia respiratoria aguda con requerimientos de ventilación mecánica y manejo en $\mathrm{UCl}$ y en casos severos la muerte.(4)

\section{PRESENTACIÓN DEL CASO}

Masculino de 37 años, sin antecedentes patológicos relevantes, quien realiza montañismo desde hace 15 años. Presenta cuadro clínico de 15 horas de evolución consistente en disnea aguda en reposo y tos seca mientras se encontraba a una altura aproximada de $4100 \mathrm{~m}$ s.n.m. escalando el Nevado del Tolima. Niega aclimatación previa y requiere iniciar el descenso, donde hay mejoría leve de disnea. En la radiografia de tórax se evidencia imagen de consolidación en pulmón derecho (Imagen A) y la tomografia de tórax es compatible con patrón de vidrio esmerilado unilateral derecho (Imagen B). Se consideró un edema pulmonar inducido por altura como primer posibilidad diagnóstica, se solicitó ecocardiograma transtorácico, fibrobroncoscopia más lavado broncoalveolar (este último descarta proceso infeccioso por microorganismos atípicos). EI ecocardiograma transtorácico reporta a nivel del septum interauricular solución de continuidad a en el foramen oval de aproximadamente $17 \mathrm{~mm}$ de diámetro con flujo de izquierda a derecha y paso de contraste salino de derecha a izquierda de gran cantidad, dilatación de cavidades derechas con insuficiencia tricuspidea grado I-II y una PSAP de $50 \mathrm{mmHg}$, hallazgos confirmados en ecocardiograma transesofágico. Se realiza cateterismo derecho en donde se identifican presiones pulmonares levemente elevadas, se realiza el cierre percutáneo del defecto con dispositivo tipo Amplatzer(B). Se dio egreso a los 7 dias con antiagregación dual con ASA y clopidogrel para 6 meses.

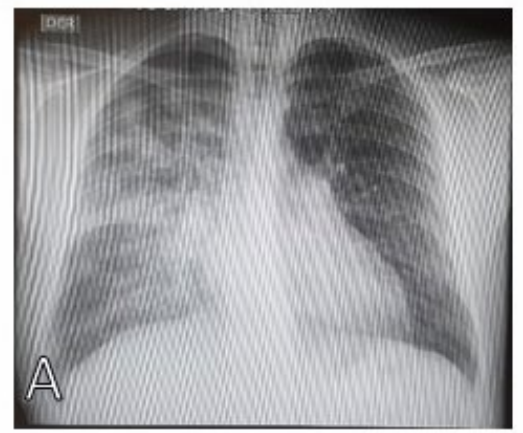

\section{DISCUSIÓN Y CONCLUSIONES}

El porcentaje de oxígeno en la atmósfera permanece constante a medida que aumenta la altitud, sin embargo, de forma inversamente proporcional hay un cambio en la presión barométrica que se refleja por una caída en el oxígeno inspirado disponible, favoreciendo la insaturación del EPGA cuando no ocurre una adecuada aclimatación(5,6). Aunque desde el punto de vista fisiopatológico es más frecuente la aparición del edema pulmonar de forma bilateral, existe un porcentaje bajo de pacientes $(2 \%)$ que presentan edema unilateral, relacionado con patologías pulmonares o cardiaca pre existentes ${ }^{(7-12)}$. Una vez instaurado el EPGA, usualmente el descenso de 300-1000 metros es el tratamiento más efectivo, acompañado de oxigenoterapia y los fármacos que disminuyen la presión de la arteria pulmonar como los calcio antagonistas. ${ }^{(13)}$ No hay evidencia para apoyar el uso de diuréticos en este escenario clínico. La corrección del defecto cardíaco como método preventivo se basa en reportes de caso (7-12). Nuestro caso reporta un paciente sano que presenta sintomatología compatible con EPGA en el lapso de 24 horas con compromiso unilateral pulmonar derecho, en quien se documenta un defecto congénito del septum interauricular desconocido previamente por el paciente y que no se habia manifestado clínicamente en ascensos a gran altura previos. Presentó adecuada evolución con manejo de soporte y tratamiento de la causa subyacente con cierre percutáneo del defecto cardiaco.

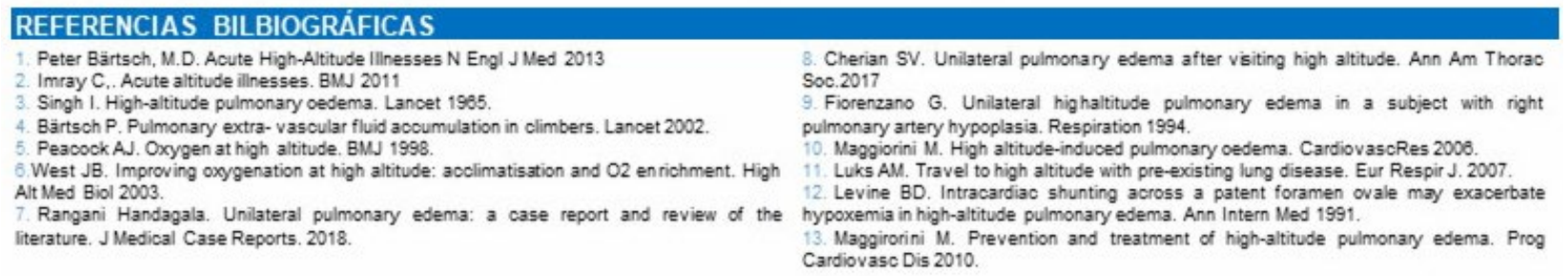

